\title{
Internationalisation Des PME De La Région Fès Meknès (Maroc) : Cartographie Des Liens Et Tendances Actuelles
}

\author{
Hamid Akdim \\ University of Maine, le Mans (France)
}

doi: 10.19044/esj.2016.v13n2p1 URL:http://dx.doi.org/10.19044/esj.2016.v13n2p1

\begin{abstract}
Globalization is not a recent phenomenon in the social sciences. The advocates argue that companies of all sizes are increasingly integrating into globalization processes. In Morocco, as in other countries, this phenomenon affects several aspects and manifests in several forms. In this paper, we analyze the impact on the mapping of links and the exchange of trade flows between firms in the Fez Meknes region (Morocco) and their foreign companies. The aim is to study the applicability of the concept of "psychic distance", particularly the importance of geographical, cultural, institutional and legislative distance in the choice of foreign partner companies.
\end{abstract}

Keywords: Internationalization, SME, Fez Meknes region, Morocco

\section{Résumé}

La mondialisation n’est pas un phénomène récent en sciences sociales. Les tenants des sciences de l'action n’ont épargné aucun effort pour l'étudier sous différents angles, spécifiquement en économie et gestion. Ils affirment tous que les entreprises, quelle que soit leur taille, ne cessent de s’intégrer de plus en plus dans des processus de mondialisation. Au Maroc, comme dans les autres pays, ce phénomène touche plusieurs aspects et se manifeste sous plusieurs formes. Dans le cadre de cet article nous analysons son impact sur la cartographie des liens et l'échange des flux commerciaux entre les entreprises de la région Fès Meknès (Maroc) et leurs clients/fournisseurs. L’objectif est d'étudier l'applicabilité de concept de « la distance psychique », notamment l'importance de la distance géographique, culturelle, institutionnelle et législative en matière des choix des entreprises partenaires.

Mots-clés: Internationalisation, PME, région Fès Meknès, Maroc 


\section{Introduction}

Depuis le début des années 1990, la mondialisation n’a pas cessé de faire l'objet d'un débat scientifique intense, portant à la fois sur sa définition et sur son impact. En effet, dans ce contexte mondialisé, on constate de plus en plus une prolifération de nouveaux acteurs internationaux autres que les Etats et les grandes multinationales. Citons par exemple les organisations internationales, les ONG, les médias, les individus, ainsi que les entreprises qui ne cessent de dépasser et contourner les frontières étatiques. Cette prolifération des acteurs a été particulièrement favorisée par l'essor de nouveaux moyens de communication et l'intensification des échanges matériels et immatériels. Cela revient à dire que la mondialisation pénètre d'une manière active dans le quotidien des entreprises et des organisations indépendamment de leur taille.

Par ailleurs, la mondialisation n'est pas un phénomène récent en sciences sociales. Les tenants des sciences de l'action n’ont épargné aucun effort pour l'étudier sous différents angles, spécifiquement en économie et gestion. Ils affirment tous que les entreprises, quelle que soit leur taille, ne cessent de s'intégrer de plus en plus dans des processus de mondialisation. Ces processus les obligent à "penser global» et à tenir compte de la mondialisation comme élément essentiel dans les différentes conceptions stratégiques. En effet, que ce soit en amont ou en aval de la chaîne de production, leurs concurrents, fournisseurs ou clients peuvent provenir de tous horizons géographiques.

Dans ces circonstances l'internationalisation se présente comme une réponse à ce processus irréversible de mondialisation pour des motifs variables selon les auteurs : optimiser l'exploitation des chaînes de valeurs (Williamson 1975 ; Buckley et Casson 1976), rechercher des avantages spécifiques (Dunning 2000), saisir les opportunités internationales via les INV ou BG (McDougall et Oviatt 1994 ; Rennie 1993), ou faire suite à la recherche de la croissance d'une manière séquentielle (Johanson et Paul 1975). A partir du moment où la décision de s'ouvrir sur des marchés étrangers est prise, la PME dispose de plusieurs choix pour opérationnaliser son projet : s'ouvrir sur des marchés géographiquement et culturellement lointains ou se limiter à des marchés partageant les mêmes spécificités que son marché domestique. La littérature a largement étudié les éléments conditionnant ces choix d'ouverture. Johanson et Vahlne (1977) avancent le concept de la distance psychique entre pays pour expliquer le développement international des entreprises. Pour ces auteurs, la distance géographique, la distance culturelle, la distance institutionnelle ou législative jouent un rôle import dans les choix des pays cibles.

L’objectif de notre recherche est d'étudier l'applicabilité de concept de « la distance » au contexte des pays du Maghreb, notamment le Maroc. Ce 
contexte présente en effet des particularités atypiques et constitue un environnement différent de ceux où ont été réalisées la plupart des recherches sur l'internationalisation des entreprises (France, Canada).

\section{Présentation de l'étude et méthodologie}

Dans toute démarche d'internationalisation, les entreprises doivent gérer « la distance » qui sépare le marché local du marché cible. Parfois, elle constitue un frein à l'expansion et au développement des entreprises, plus particulièrement les PME lesquelles se caractérisant par une faiblesse de ressources, humaines, financières et technologiques. Nous avons mené une enquête auprès des entreprises de la région de Fès Meknès, afin, d’analyser la validité de cette hypothèse aux entreprises étudiées, et, comprendre comment les PME dépassent ces risques de distance (langues, cultures, politiques et économiques...).

L’industrie de la région de Fès-Meknès se compose de 885 unités de production, dont 229 du secteur textile et 272 de l'agroalimentaire, répartie dans les deux régions selon le tableau 1 :

Tableau $1:$ nombre des entreprises d'IIA et d'ITC dans la région de Fès Meknès ${ }^{1}$

\begin{tabular}{|c|c|c|c|}
\hline Nom de la région & IAA & ITC & Total \\
\hline FES-BOULMANE & 160 & 245 & 405 \\
\hline MEKNES-TAFILALET & 69 & 27 & 96 \\
\hline Total & 229 & 272 & 501 \\
\hline
\end{tabular}

Si le tissu industriel des secteurs de textile et de l'agroalimentaire de Fès-Meknès est composé de 501 entreprises, avec un taux de sondage de $13 \%$ ou de 14\%, notre échantillon doit contenir entre 65 et 70 entreprises. Les questionnaires ont été administrés séparément dans les deux secteurs de l'agroalimentaire et du textile sur un échantillon de 68 entreprises (38 dans le textile et 30 dans l'agroalimentaire). Nous avons profité du Salon de l'agriculture et de l'agroalimentaire qui a eu lieu à Meknès entre le 28 avril et 3 mai 2015, pour collecter une partie des observations. Le reste des questionnaires a été administré directement en contactant les sièges des entreprises. Le relationnel reste la formule pratique qui nous a permis de résoudre le problème de la réticence des professionnels à s’impliquer dans notre étude. Parmi les réseaux mobilisés, on cite à titre d'exemple les services de l'Office national de sécurité sanitaire des produits alimentaires (ONSSA) et l'agence commerciale du pôle fret de l'ONCF. Le questionnaire que nous avons élaboré comprend sept sections :

1 IAA : Industrie agroalimentaire/ ITC : Industrie textiles et cuir

Source des données : Publication du Haut-Commissariat au Plan du Maroc, Monographies régionales 2011. 
Section 1 : caractéristiques de l'entreprise,

Section 2 : exportations,

Section 3 : importations,

Section 4 : sous-traitance,

Section 5 : production et innovation,

Section 6 : modalités d'internationalisation,

Section 7 : renseignements complémentaires sur l'entreprise,

\section{Résultats}

\section{Entreprises étudiées : quelques faits caractéristiques}

A la lecture des résultats illustrés sur la figure 1 on constate que $49 \%$ des entreprises étudiées traitent les affaires avec des grandes entreprises étrangères. Tandis que 21\% traitent avec des grandes entreprises marocaines. $18 \%$ des entreprises interviewées collaborent avec des PME étrangères, alors que seulement $12 \%$ coopèrent avec des PME marocaines. On constate une dominance des grandes entreprises dans le portefeuille des PME étudiées.

$\square$ PMEMA $\square$ PMEETR $\square$ GEMA $\square$ GEETR

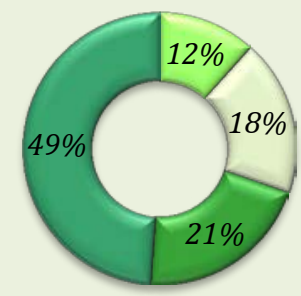

Figure 1: principal client de l'entreprise

$\square$ Avant $1980 \square$ 1981-1990 $\square$ 1991-2000 $\square 2001-2014$

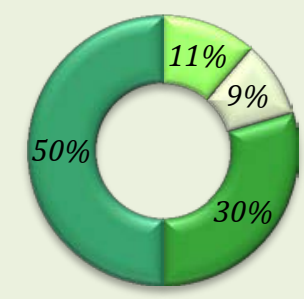

Figure 2 : année de création de l’entreprise 
Vu les difficultés et les exigences de l'action à l'international, et sur la base de l'échantillon traité dans la région de Fès - Meknès, on peut donc considérer qu'un effort notable a été fait par l'entreprise marocaine pour s'inscrire dans la dynamique internationale des échanges. Son insertion dans ce marché révèle un certain niveau de compétence et de performance, aussi bien dans les PME que pour les TPE. Mais une certaine organisation s'impose pour réussir le défi de l'expansion au-delà du marché domestique, ainsi qu'une vision et une intelligence économique permettant d'assimiler les exigences de la coopération à l'international. C'est une conscience qui se développe au fil des années.

En analysant l'évolution de la création des entreprises composant l'échantillon de notre étude, on s'aperçoit que la dernière décennie (2001 2014) a connu une dynamique plus intense de création, comparée avec les périodes antérieures. La figure 2 montre que $50 \%$ des entreprises ont été créées entre 2001 et 2014. Alors que 30 \% des entreprises contactées ont été créées entre 1991 et 2000. Les entreprises créées entre 1981 et 1990 représentent 9\%. Le reste des entreprises (soit $11 \%$ ) ont été créées avant 1980. Il se dégage de ce constat que le processus de création d'entreprises ouvertes à l'international s'intensifie au cours des dernières années. Cela pourrait se traduire par les efforts des autorités, pour encourager l'ouverture internationale de l'économie marocaine. Ces efforts, s'illustrent par plusieurs programmes et coopérations engagées récemment, on peut citer à titre d'illustration :

- L'accord du libre-échange Maroc-USA, entré en vigueur en 2006.

- Le statut avancé Maroc-Union Européenne en 2008.

- Plusieurs accords de partenariat bilatéral et de coopération décentralisée entre le Maroc et d'autres pays sur les cinq continents.

- Des mesures institutionnelles, financières et fiscales encourageantes.

Cette volonté politique s'accompagne d'une volonté des entreprises de profiter des opportunités qu'offre ce contexte. Ce qui implique un impact positif sur l'entreprise en termes de croissance, d'investissement et de développement, mais également sur la masse salariale. Comme illustré sur la figure 3 , on voit que $18 \%$ des entreprises engagent plus de 300 salariés. $7 \%$ d'entre elles ont entre 301 et 400 salariés et $11 \%$ entre 401 et 550 salariés ; $27 \%$ ont moins de 50 salariés ; $16 \%$ des entreprises ont entre 51 et 100 salariés ; $16 \%$ entre 101 et 200 salariés et enfin 23\% entre 201 et 300 salariés. 


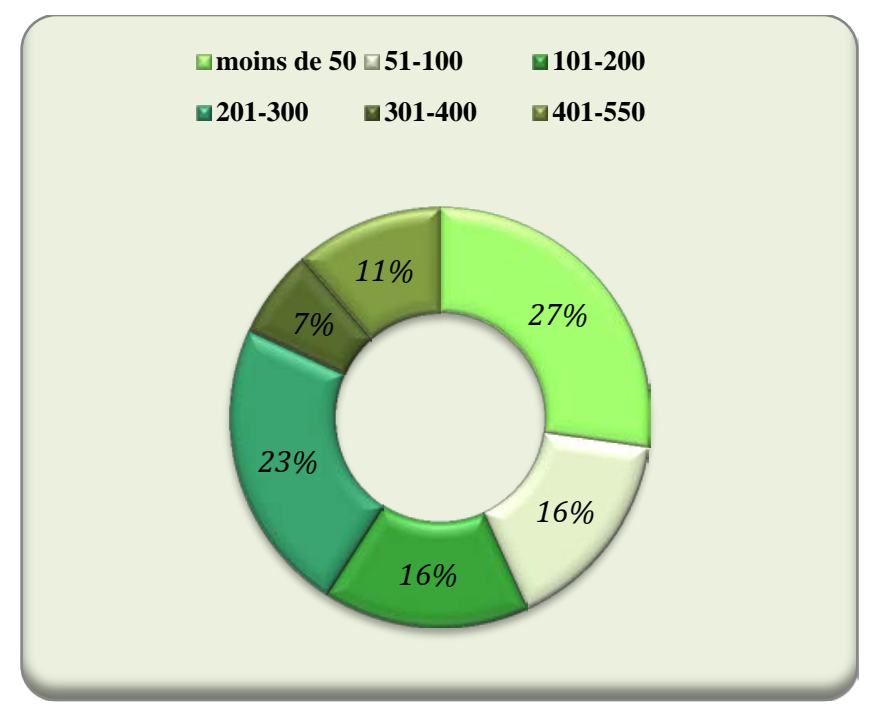

Figure 3 : nombre d'employés dans l'entreprise

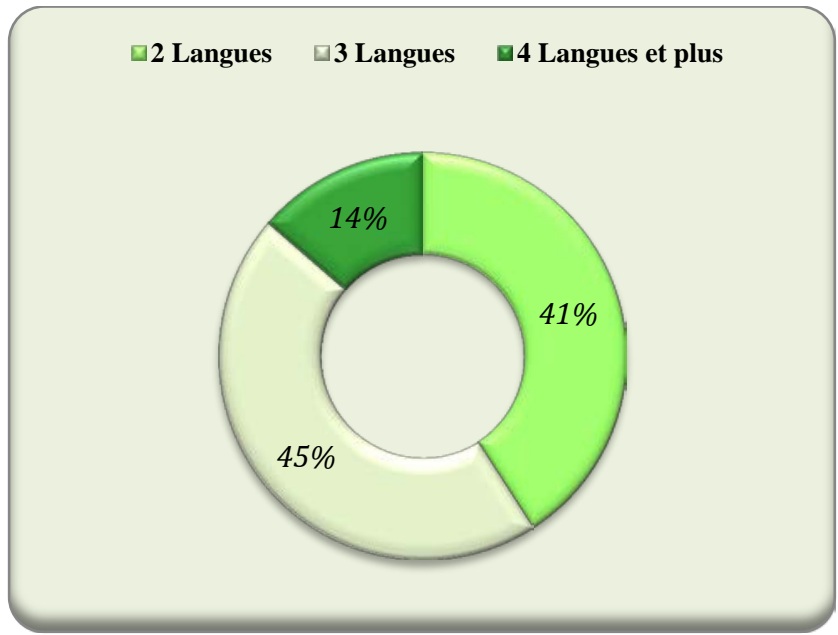

Figure 4 : nombre de langues parlées dans l'entreprise

Il est certain que l'intérêt à l'internationalisation, observé aussi bien chez les PME du secteur de textile que dans les PME du secteur agroalimentaire, traduit une dynamique motivée, qui se rattache aux incitations variées, relatives à l'entreprise et son organisation, au chef d'entreprise, mais aussi aux nouveaux modes d'échanges et de communication qui ne cessent de se diversifier. L'organisation et le dynamisme des entreprises étudiées seront discutés dans les chapitres suivants. Pour ce qui est des aspects relatifs à la communication, le nombre de langues étrangères parlées au sein de l'entreprise est un indicateur qui illustre, même partiellement, son degré de préparation pour faire face aux exigences de l'internationalisation. En effet, l'échange permanent avec 
l'étranger, la compréhension des besoins et des exigences de l'autre ainsi que les écarts interculturels imposent un certain niveau de la maîtrise des langues au sein de l'entreprise. La figure 5.5 montre que $41 \%$ des entreprises de l'échantillon annoncent deux langues étrangères parlées. $45 \%$ des entreprises signalent 3 langues étrangères parlées et $14 \%$ déclarent 4 langues étrangères parlées. La langue française figure dans la majorité des cas. L'anglais vient en deuxième position, même avec des degrés de maîtrise parfois élémentaires. D'autres langues comme l'espagnol, l'italien et l'allemand viennent en dernière position. Le chinois et le russe sont négligés par les professionnels composant le champ de l'étude. Une plus ample ouverture à la fois sur l'anglais et sur les autres langues étrangères est donc nécessaire à moyen et long terme, pour que l'entreprise marocaine puisse profiter du réseautage mondial et des opportunités éventuelles des grands foyers démographiques et des nouveaux centres d'affaires émergents ailleurs.

\section{Géographie des liens et secteurs dominants}

Notre choix a depuis le début, porté sur deux secteurs clés de l'économie marocaine et de la région Fès - Meknès : le textile et l'agroalimentaire. Le premier est connu pour son passé pionnier, mais actuellement il souffre de contraintes structurelles et affronte sur la scène internationale une féroce concurrence. Le second s'impose en raison des orientations stratégiques du Maroc dans ce domaine, et au vu des potentialités régionales de son développement. Les résultats de l'enquête réalisée sur les entreprises actives à l'international dans ces deux secteurs à l'échelle de la région Fès Meknès, sont présentés dans les paragraphes ci-dessous. La figure 5 montre que 59 \% des entreprises enquêtées sont dans le textile et $41 \%$ sont des entreprises de l'agroalimentaire.

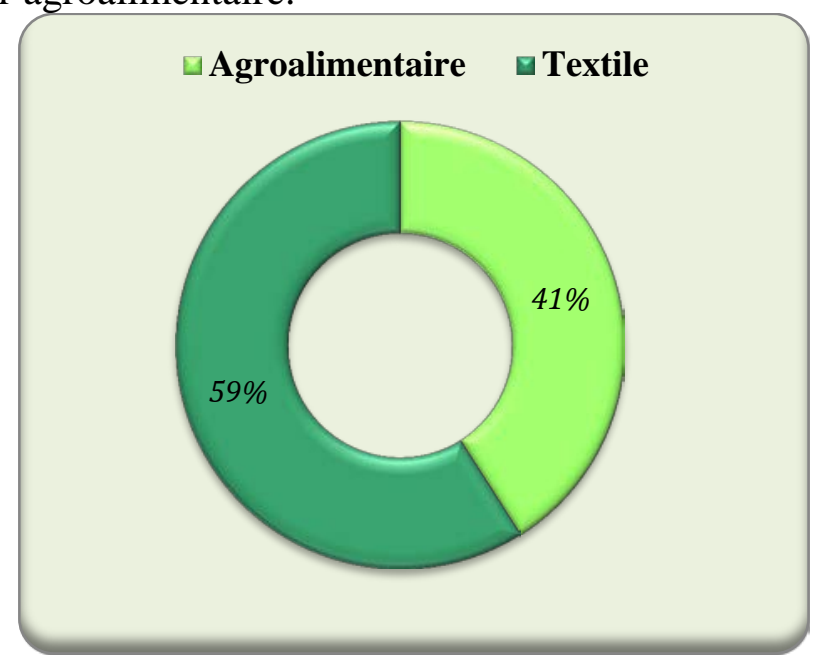

Figure 5 : Secteur d'activité 
La résilience du secteur du textile et le renforcement du lancement de l'agroalimentaire sont conditionnés par de nombreux facteurs liés notamment à la qualité du produit, au coût de production, au dynamisme managérial, à la gouvernance et à l'organisation de l'entreprise, que nous ciblons dans cette analyse. Mais on peut ajouter à ces facteurs l'attractivité de certains marchés et aires géographiques, soit pour leurs effets de proximité ou leur environnement favorable.

La figure 6 montre les aires géographiques connaissant des grands flux d'exportation des produits du textile et de l'agroalimentaire par les entreprises de la région Fès - Meknès. On y observe une diversification assez importante puisque des transactions ont été faites avec des partenaires dans plusieurs continents (Europe, Amérique, Asie). L'intensité des liens diffère certes, mais cet effort de diversification ne passera pas inaperçu. Comme on le constate sur la figure 6, l'effet de proximité géographique, culturel et historique reste fort sur la répartition géographique des exportations. On voit sur la carte que la majorité des liens est tissée avec l'Europe.

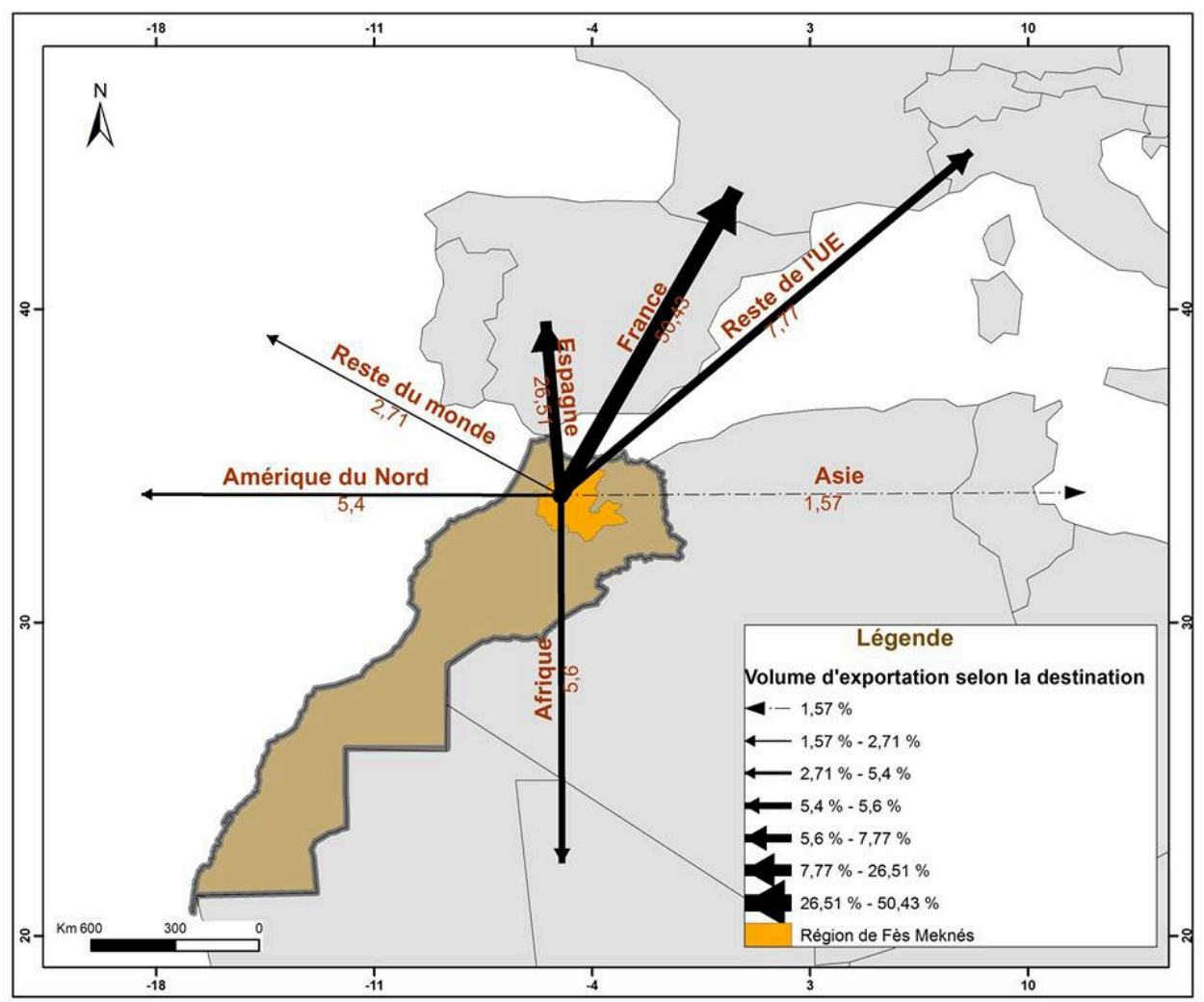

Figure 6 : répartition géographique des exportations de secteurs textiles/agroalimentaire de la région de Fès- Meknès ${ }^{2}$

${ }^{2}$ Source des données : enquête de terrain, Avril 2015 
Conformément aux orientations géographiques et à la tendance des échanges internationaux à l'échelle du Maroc, la Région Fès - Meknès privilégie les échanges avec l'Europe et au sein de l’UE avec la France. La moitié des transactions se font sur cet axe avec 50,43\% des exportations de la région de Fès - Meknès. L'Espagne vient en deuxième position avec 26,51 \% des exportations. Le reste de l'Europe représente $7.77 \%$ des exportations de la région. La destination africaine est représentée dans ces échanges avec une proportion de $5.6 \%$ des exportations de la région. Ces marchés sont toujours porteurs à condition de rendre plus performants les modes d'action à l'international et de préparer les entreprises sur le plan organisationnel pour répondre aux exigences des marchés étrangers.

En posant le curseur d'analyse sur les importations, on voit que les principaux fournisseurs de la région Fès - Meknès dans le secteur du textile et de l'agroalimentaire sont essentiellement les pays d'Europe et d'Asie du Sud -Est comme le montre la figure 7.

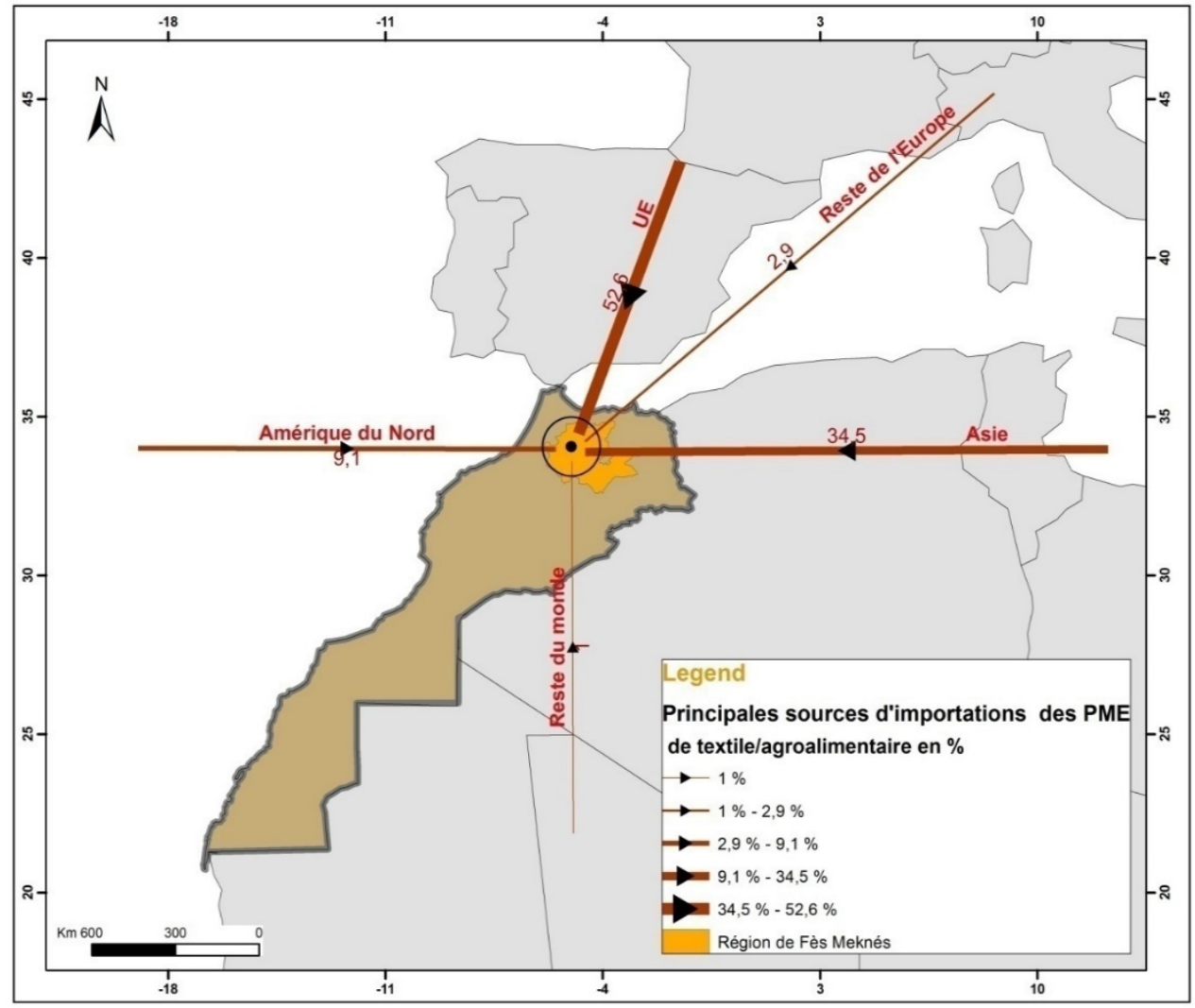

Figure 7 : répartition géographique des sources d'importation des entreprises de la région de Fès Meknès ${ }^{3}$

${ }^{3}$ Source des données : enquête de terrain, Avril 2015 
L’Union Européenne est la première source des importations vers la région, avec une fréquence de 52\%. L’Asie du Sud - Est, est la deuxième source des importations avec une fréquence de 34,5\%. Dans les deux cas, l'importation concerne les équipements et certains produits intermédiaires exploités dans la production.

Ces résultats montrent que les PME par rapport aux grandes entreprises ont plus de difficulté à contourner la distance géographique, culturelle et législative entre le pays d'origine et les marchés cibles. Ces résultats corroborent avec ceux de Johanson et Vahlne (1977); Mayrhofer (2004) et Vachani (2005). En effet, les entreprises décidant d'internationaliser leurs activités doivent faire face à un nouvel environnement, à un nouveau contexte et une nouvelle règlementation. Pour réussir au mieux leur internationalisation les entreprises étudiées choisissent généralement les marchés proches au marché domestique, dont elles disposent d'informations pratiques sur la réglementation en vigueur, la politique économique, les codes socioculturelles et l'intensité concurrentielle.

Les résultats de cette recherche s'inscrivent dans la même ligne que les résultats d'autres recherches qui ont étudié le concept de la distance et son impact sur l'internationalisation des entreprises (Bilkey et Tesar (1977), Johanson et Vahlne (1977), Johanson et Wiedersheim-Paul (1975). Ces recherches mettent en avant la progressivité du développement international des entreprises. La distance s'aggrave en fonction du risque perçu lié à la langue, la culture, l'éloignement géographique et à la stabilité politique.

Par ailleurs, on constate que les échanges des PME étudiées avec les pays africains restent modestes et ne répondent pas aux attentes des orientations politiques et des efforts déployés ces dernières années. Est-il nécessaire de rappeler l'enracinement africain du Maroc et l'importance des efforts déployés depuis l'indépendance pour réaffirmer cette position. Comme disait Hassan 2 « Le Maroc est un arbre dont les racines plongent en Afrique et les branches s'étendent en Europe ", Aujourd'hui ces racines se développent à triple vitesse : croissance, ramification et différenciation. En effet, la stratégie de coopération Sud-Sud et les nouveaux mécanismes de partenariat mise en œuvre depuis les années 2000 en témoigne. De Dakar à Addis-Abeba en passant par Abidjan, Libreville, Abuja, Kigali....SM le roi Mohamed 6 multiplie les visites et les déplacements en Afrique pour instaurer une nouvelle démarche innovante et ambitieuse. Néanmoins ces plans d'actions sont exploités par les grandes entreprises et les grands groupes, il serait intéressant de réfléchir à une stratégie impliquant les PME. 


\section{Conclusion}

L’analyse des résultats de cette recherche révèle une diversification assez importante des zones avec lesquelles se développent les échanges des entreprises de la région Fès-Meknès. Mais avec une dominance des destinations traditionnelles comme la France et l'Espagne. Ceux-ci illustrent l’importance de la proximité "psychologique" dans les choix des partenaires aussi bien en import qu'à l'export. L'effort cumulatif à investir pour des visées lointaines reste donc considérable. Plus particulièrement vers les pays africains, afin de s’aligner avec la stratégie politique et les efforts déployés ses dernières années pour encourager une coopération Sud/Sud.

La question de la distance s’impose dans tout essai de développement à l'internationalisation. En effet, le développement international confronte les entreprises étudiées aux problèmes de la distance qui sépare le marché domestique des marchés potentiels. Des problèmes qui peuvent être liés aux écarts politiques, culturels et sociaux.

Néanmoins, malgré ces contributions, le présent travail affronte quelques limites qu'il convient d’énumérer, pour éclairer les recherches futures et ouvrir des pistes potentielles d'investigation à explorer. Notre étude s'est limitée aux entreprises de secteur de textile et agroalimentaire, dans une perspective de généralisation des résultats, il convient d’élargir le champ d'étude à d'autres secteurs pionniers dans la région comme l'artisanat ou certaines activités de services.

\section{References:}

1. Buckley, P. J., \& Casson, M. C. (1976). The future of multinational enterprise Macmillan: London.

2. Dunning, J. H. (2000). 5 The eclectic paradigm of international production. The nature of the transnational firm, 119.

3. Geringer, J. M., Tallman, S., \& Olsen, D. M. (2000). Product and international diversification among Japanese multinational firms. Strategic Management Journal, 21(1), 51-80.

4. Gomes, L., \& Ramaswamy, K. (1999). An empirical examination of the form of the relationship between multinationality and performance. Journal of international business studies, 30(1), 173187.

5. Hennart, J. F. (2011). A theoretical assessment of the empirical literature on the impact of multinationality on performance. Global Strategy Journal, 1(1-2), 135-151.

6. Johanson, J., \& Wiedersheim-Paul, F. (1975). The internationalization of the firm-four swedish cases 1 . Journal of management studies, 12(3), 305-323. 
7. Mayrhofer, U. (2004), « International Market Entry: Does the Home Country Affect Entry Modes Decisions? », Journal of International Marketing, 12: 4, 71-96.

8. Maskini, N., \& Maa, F. (2016). La compétitivité des entreprises exportatrices et les contraintes de financement: cas du Maroc/[Business competitiveness and export financing constraints: case of Morocco]. International Journal of Innovation and Applied Studies, 17(2), 464.

9. McDougall, P. P., \& Oviatt, B. M. (2000). International entrepreneurship: the intersection of two research paths. Academy of management Journal, 43(5), 902-906.

10. McDougall, P. P., Shane, S., \& Oviatt, B. M. (1994). Explaining the formation of international new ventures: The limits of theories from international business research. Journal of business venturing, 9(6), 469-487.

11. Rainelli, M. (2015). Les grandes théories du commerce international. Repères (11), 45-80.

12. Rennie, M. W. (1993). Born global. The McKinsey Quarterly, (4), 45-53.

13. Tallman, S., \& Li, J. (1996). Effects of international diversity and product diversity on the performance of multinational firms. Academy of Management journal, 39(1), 179-196.

14. Thomas, D. E., \& Eden, L. (2004). What is the shape of the multinationality-performance relationship?. Multinational Business Review, 12(1), 89-110.

15. Vachani, S. (2005), «Problems of foreign subsidiaries of SMEs compared with large companies ", International Business Review, 14, 415-439.

16. Williamson, O.E. (1975). Market and Hierarchies. Analysis and Antitrust Implications, the Free Press. New York. 